\title{
SEMIGROUPS OVER GENERALIZED TREES
}

\author{
T. E. HAYS \\ (Received 23 February 1977) \\ Communicated by T. E. Hall
}

\begin{abstract}
A semigroup over a generalized tree, denoted by the term $\mathscr{H} \mathscr{L}$-semigroup, is a compact semigroup $S$ such that Green's relation $\mathscr{H}$ is a congruence on $S$ and $S / \mathscr{H}$ is an abelian generalized tree with idempotent endpoints and $E(S / \mathscr{H})$ a Lawson semilattice. Each such semigroup is characterized as being constructible from cylindrical subsemigroups of $S$ and the generalized tree $S / \mathscr{H}$ in a manner similar to the construction of semigroups over trees and of the hormos. Indeed, semigroups over trees are shown to be particular examples of the construction given herein.
\end{abstract}

Subject classification (Amer. Math. Soc. (MOS) 1970): primary 22 A 15;

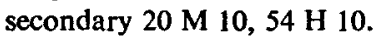

Keywords: compact semigroup, generalized tree, semigroup over a tree, Lawson semilattice, inverse limit preserving functor.

\section{Introduction}

Mislove $(1969,1974)$ defines a compact semigroup $S$ to be a semigroup over a tree if Green's $\mathscr{H}$-relation is a congruence on $S$ and $S / \mathscr{H}$ is an abelian tree with idempotent endpoints. He then proceeds to characterize completely the semigroup $S$ in terms of $S / \mathscr{H}$ and certain cylindrical subsemigroups of $S$.

Our purpose here is to generalize this result by obtaining a similar characterization of those compact semigroups $S$ with $S / \mathscr{H}$ an abelian generalized tree with idempotent endpoints and $E(S / \mathscr{H})$ a Lawson semilattice. As is to be expected in an undertaking of this nature, several of the basic techniques used by Mislove are, with modification, applicable to this more general situation. While new formulations and arguments must be given as the paper proceeds, we will follow the basic pattern established by Mislove. We will present the information however, in the much neater categorical approach developed by Bowman (1971).

The notation and terminology will be that of Hofmann and Mostert (1966). Along with this, Kelley (1955) and Mitchell (1965) will serve as our standard references. This work forms a portion of the author's doctoral dissertation and he wishes to express his gratitude and appreciation to Professor J. H. Carruth for his patience and advice during its preparation. 


\section{Preliminaries}

Throughout this paper the term "semigroup" will always mean topological semigroup.

Definition 2.1. A continuum is hereditarily unicoherent if the intersection of any two subcontinua is connected. A generalized tree is a hereditarily unicoherent continuum on which there exists a closed monotone partial order with unique minimal element (Koch and Krule, 1960).

The structure of generalized trees has been studied by Koch and Krule (1960) and Ward $(1954,1957,1958)$. We now list some of the properties established in these works, and shall use these properties throughout this work without specific reference.

If $a, b \in X$, a generalized tree, there is a unique arc in $X$ from $a$ to $b$, denoted by $[a, b]$. Each subcontinuum of a generalized tree is itself a generalized tree (Ward, 1957), and if $f: X \rightarrow X$ is a continuous function, there is an $x$ in $X$ with $f(x)=x$; that is, generalized trees possess the fixed point property (Ward, 1957).

Definition 2.2. A point $x \in C$, a continuum, is a weak cutpoint of $C$ if there are $y, z$ in $C \backslash\{x\}$ such that any subcontinuum of $C$ containing $y$ and $z$ also contains $x$.

Definition 2.3. A point $x$ of an arc-wise connected continuum $X$ is an endpoint of $X$ if it separates no $\operatorname{arc}$ in $X$.

Lemma 2.4. Let $T$ be a generalized tree. If $x$ is not a weak cutpoint of $T$, then $x$ is an endpoint of $T$.

Proof. Let $A$ be an arc in $T$ with endpoints $a$ and $b$. Let $x$ separate $A$. Then $x \in A$ with $a \neq x$ and $b \neq x$. If $C$ is a subcontinuum of $T$ containing $a$ and $b$, then $A \cap C$ is a subcontinuum of the $\operatorname{arc} A$ containing $a$ and $b$. Thus $A \cap C=A$ and $x \in C$. As $C$ was an arbitrary continuum containing $a$ and $b, x$ is a weak cutpoint of $T$. The result then follows by contraposition.

We now proceed to establish some results about semigroups whose underlying space is a generalized tree. In several cases they are extensions of Mislove's work with semigroups whose underlying space is a tree. In most of what follows we will be concerned with semigroups on generalized trees with idempotent endpoints and commuting idempotents. The following result shows that we can assume that the generalized trees with which we work are abelian.

LEMMA 2.5 (Hunter, 1959). Suppose $T$ is a semigroup on a hereditarily unicoherent arc-wise connected continuum. If the endpoints of $T$ are idempotent and commute, one with another, then $T$ is abelian. 
Lemma 2.6. Suppose $T$ is a semigroup on a generalized tree with idempotent endpoints in which the idempotents commute. Then the maximal subgroups of $T$ are totally disconnected and hence $T$ has a zero.

Proof. Let $e \in E(T)$. Then $C_{H(e)}(e)$, the identity component of $H(e)$, is a compact connected subgroup of $T$, and so it is a generalized tree. As $C_{H(e)}(e)$ then has the fixed point property, $C_{H(e)}(e)=\{e\}$ and $H(e)$ is totally disconnected.

If $e \in E(T) \cap M(T), H(e)=e T e$ is connected and totally disconnected, whence $H(e)$ is trivial. Thus $M(T) \subseteq E(T)$ and, hence, $M(T)$ is a point since $E(T)$ is abelian.

Lemma 2.7. Suppose $T$ is an abelian semigroup on a generalized tree with idempotent endpoints. Then $[0, e]$ is a standard thread and $H(e)=\{e\}$ for each $e \in T$.

Proof. Fix $e \in E(T)$. According to Lemma 2.6, $H(e)$ is totally disconnected for each $e \in E(T)$. Thus by Exercise 8 of Hofmann and Mostert (1966), p. 159 there is a standard thread $I$ running from $e$ to 0 . But $[0, e]$ is the unique arc from $a$ to 0 in $T$, and so $I=[0, e]$.

Since $T$ has idempotent endpoints, $T=\bigcup\{[0, f]: f \in E(T)\}$, and so

$$
e T e=\bigcup\{[0, e f]: f \in E(T)\} .
$$

Since $e T e$ is a generalized tree with idempotent endpoints and no point of $H(e)$ can be a weak cutpoint of $e T e$ (see Hunter, 1961, and Koch, 1957), we must have $H(e)=\{e\}$ by Lemma 2.4 .

Definition 2.8. Let $T$ be an abelian semigroup on a generalized tree with idempotent endpoints and $X=E(T)$. We define $X^{\prime}=\{x \in X \backslash\{0\}: x$ is isolated in $[0, x] \cap X\}$ and $x^{\prime}=\sup ([0, x) \cap X)$ if $x \in X^{\prime}$.

We conclude this section with the establishment of some convergence properties in $T$.

Proposition 2.9. Let $T$ be an abelian semigroup on a generalized tree with idempotent endpoints, $\left\{x_{i}\right\}_{i \in I}$ a net in $X$ converging to $x, x \in X^{\prime}$ with $x_{i} x=x_{i}$ for each $i \in I$. Then for a residual subset $J \subseteq I, x_{i} \in X^{\prime}, x^{\prime} x_{i}=x_{i}^{\prime}$ for each $i \in J$ and the net $\left\{x_{i}^{\prime}\right\}_{i \in J}$ converges to $x^{\prime}$.

Proof. Since $\left\{x_{i}\right\}_{i \in I}$ converges to $x$, and $x x^{\prime}=x^{\prime}$ by Lemma 2.7 , the net $\left\{x^{\prime} x_{i}\right\}_{i \in I}$ converges to $x^{\prime}$ by the continuity of multiplication. Hence there is an element $j \in I$ such that $i \geqslant j$ implies $x^{\prime} x_{i} \neq x_{i}$. Let $J=\{i \in I: i \geqslant j\}$ and let $i \in J$. Then by Phillips (1963), $\left[x^{\prime}, x x_{i}\right]=\left[x^{\prime} x_{i}, x_{i}\right]$. Since $T$ is abelian, translation by $x_{i}$ is a homomorphism and $\left(x^{\prime}, x\right) \cap X=\square$ implies by Cohen and Krule (1959) that $\left(x^{\prime} x_{i}, x_{i}\right) \cap X=\square$. Since $\left[x^{\prime} x_{i}, x_{i}\right] \subseteq\left[0, x_{i}\right], x_{i} \in X^{\prime}$ and $x_{i}^{\prime}=x^{\prime} x_{i}$. Clearly the net $\left\{x_{i}^{\prime}\right\}_{i \in J}$ converges to $x^{\prime}$. 
The following proposition was proved by Mislove $(1974 ; 1969$, p. 79$)$ for trees, but his proof is also valid in the more general setting and hence we content ourselves with the statement of the more general proposition.

Proposition 2.10. Let $T$ be an abelian semigroup on a generalized tree and $X=E(T)$. If $x \in X^{\prime}$ and $x$ is not isolated in $x X$, then $D=\{y \in X: x y=y$ and $y$ isolated in $y X\}$ is a directed set under $y \leqslant z$ if and only if $y z=y$. Furthermore, the net $\{y\}_{y \in D}$ converges to $x$.

LEMMA 2.11. Let $T$ be an abelian semigroup on a generalized tree with idempotent endpoints. If $\left\{x_{i}\right\}_{i_{\epsilon} I}$ converges to $x$ with $x_{i} x=x_{i}$ for each $i \in I$, then $\left[0, x_{i}\right]$ converges in the lim sup-lim inf sense to $[0, x]$. Moreover, if $x_{i} \in X^{\prime}, x \in X^{\prime}$ with $x_{i} x^{\prime}=x_{i}^{\prime}$, then $\left[x_{i}^{\prime}, x\right]$ converges to $\left[x^{\prime}, x\right]$.

Proof. By Phillips (1963), $[0, x] x_{i}=\left[0, x_{i}\right]$. It is easy to see that $[0, x] x_{i}$ converges to $[0, x] x=[0, x]$. Hence $\left[0, x_{i}\right]$ converges to $[0, x]$. A similar argument shows that $\left[x_{i}^{\prime}, x_{i}\right]$ will converge to $\left[x^{\prime}, x\right]$.

\section{A generalization of semigroups over trees}

In this section we prove the first major result of the paper, a generalization of Mislove's construction of semigroups over trees. In doing this we resort to the convenient language of category theory with Mitchell (1965) as our basic reference.

Notation. A semilattice $X$ will be considered a category throughout this paper by letting the elements of $X$ be the objects and defining $\operatorname{Hom}(y, x)$ to be singleton if $y x=x$ and the empty set otherwise. The unique morphism from $y$ to $x$ will be denoted by $y \subset x$. Throughout the remainder of the paper $\mathscr{C}$ will denote the category of cylindrical semigroups and continuous homomorphisms and $\mathscr{M}$ will denote the category of surmorphisms whose domains and ranges are objects of $\mathscr{C}$ and whose morphisms are ordered pairs of homomorphisms such that

$$
\left(h_{1}, h_{2}\right) \in \operatorname{Hom}(f, g)
$$

if and only if the following diagram has meaning and commutes:

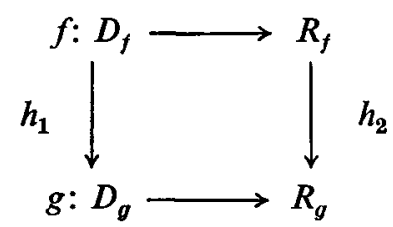

where $D_{f}$ is the domain of $f$ and $R_{f}$ the range of $f$. 
Definition 3.1. A Lawson semilattice is a semilattice whose topology has a basis of subsemilattices.

We now come to the generalization of the concept of generalized collection (see Mislove, $1974 ; 1969$, p. 80). In fact with the exception of two significant additions, this is the reformulation of that concept in terms of the language of category theory.

Definition 3.2. The ordered pair $(T, F)$ is a generalized pair if $T$ is an abelian generalized tree with idempotent endpoints, $X=E(T)$ is a Lawson semilattice, and $F$ is a functor from $X$ into the product category $\mathscr{C} \times \mathscr{M}$ which satisfies the following:

(a) $\Pi_{\mathscr{C}} F$ is inverse limit preserving from $X$ into $\mathscr{C}$.

(b) Letting $S_{x}=\Pi_{\mathscr{C}} F(x), n_{x}=\Pi_{\mathscr{M}} F(x), m_{x y}=\Pi_{\mathscr{C}} F(y \subset x)$, then $S_{x}, m_{x y}$, and $n_{x}$ satisfy the following:

(i) $S_{x}$ is the domain of $n_{x}$ for each $x \in X$ and $n_{x}(s)=n_{x}(t)$ if and only if $s$ and $t$ are $\mathscr{H}$-related in $S_{x}$.

(ii) If $x \notin X^{\prime}$ then $S_{x}=H\left(1_{x}\right)=M_{x}$ is a group and $n_{x}\left(S_{x}\right)=\{x\}$.

(iii) If $x \in X^{\prime}$ then $n_{x}\left(S_{x}\right)=\left[x^{\prime}, x\right]$.

(iv) If $x \neq y$ then $S_{x} \cap S_{y}=\square$.

(v) If $x \in X^{\prime}$ then $m_{x^{\prime} x} \mid M_{x}$ is an injection, where $M_{x}$ is the minimal ideal of $S_{x}$.

(vi) If $x \in[0, y), m_{x y}\left(S_{y}\right) \subseteq H\left(1_{x}\right)$.

(vii) If $x, y \in X^{\prime}, x y=x$ and $x^{\prime}=y^{\prime}$, then $m_{x y} \mid n_{y}^{-1}\left[y^{\prime}, t\right]$ is an injection into $n_{x}^{-1}\left[x^{\prime}, t\right]$, where $t=\sup \left(\left[x^{\prime}, x\right] \cap\left[y^{\prime}, y\right]\right)$.

(viii) If $x, y \in X$ with $x y=x$, then $s \in S_{y}$ implies $n_{x}\left(m_{x y}(s)\right)=x n_{y}(s)$.

Notation. If $(T, F)$ is a generalized pair, let $S^{\prime}=\bigcup\left\{S_{x}: x \in X\right\}$ and $p: S^{\prime} \rightarrow X$ be defined by $p(s)=x$ if and only if $s \in S_{x}$.

The following proposition indicates how a compact semigroup may be constructed utilizing a generalized pair. It follows immediately from the definition of a generalized pair and Theorem 1.3 of Bowman (1971).

Proposition 3.3. Let $(T, F)$ be a generalized pair and $S^{\prime}=\bigcup\left\{S_{x} ; x \in X\right\}$.

(A) For $s, t \in S^{\prime}$, define $s t=m_{v p(s)}(s) m_{v p(t)}(t)$ where $v=p(s) p(t)$. With this multiplication $S^{\prime}$ is an algebraic semigroup.

(B) For $U$ open in $X, V$ open in $S_{u}$ where $u$ is zero of the compact semilattice generated by $U$, define $W(U, V)=p^{-1}(U) \cap\left\{s \in S^{\prime}: m_{u p(s)}(s) \in V\right\}$. The collection of $W(U, V)$ forms a basis of a topology on $S^{\prime}$ relative to which $S^{\prime}$ is a compact semigroup.

The proof of the following proposition is straightforward and is omitted. 
Proposition 3.4. Let everything be as in Proposition 3.3. For each $x \in X$, the topology induced on $S_{x}$ as a subset of $S^{\prime}$ is the same as its original topology.

Proposition 3.5. Let everything be as in Proposition 3.3. Then $p: S^{\prime} \rightarrow X$ and $n: S^{\prime} \rightarrow T$ defined by $n(s)=n_{p(s)}(s)$ are continuous surmorphisms of $S^{\prime}$ onto $X$ and $T$, respectively.

Proof. The proof that $p$ is a continuous surmorphism is straightforward. The proof by Mislove $(1974 ; 1969$, p. 94), that $n$ is an algebraic surmorphism is still valid in this setting and so we concern ourselves with showing the continuity of $n$. We do this by a net argument.

Let $\left\{s_{i}\right\}_{i_{\in} I}$ converge to $s$ in $S^{\prime}$. Since $p$ is continuous, $\left\{p\left(s_{i}\right)\right\}_{i_{\in} I}$ converges to $p(s)=x$. Let $x_{i}=p\left(s_{i}\right)$ and $y_{i}=p\left(s_{i}\right) p(s)$. By passing to subnets if necessary we have one of the following: $y_{i}=x$ for each $i \in I$ or $y_{i} \neq x$ for each $i \in I$. Let $B=\{U$ : $U$ is an open semilattice containing $x\}$ be directed by $U \leqslant V$ if and only if $V \subseteq U$. In the former case define the net $\left\{x_{U}\right\}_{U \in B}$ by $x_{U}=x$ for each $U \in B$, and in the latter case let $x_{U}$ be the zero of $U^{*}$, where * denotes closure, for each $U \in B$. Note that in either case the net $\left\{x_{U}\right\}_{U \in B}$ has the property $x_{U} x=x_{U}$ and $x_{U} x_{V}=x_{U}$ if $U \leqslant V$. Since $\left\{x_{i}\right\}_{i_{\epsilon} I}$ converges to $x$, we have for $U \in B$, the set $\left\{i \in I: x_{U} x_{i}=x_{U}\right\}$ is residual in $I$. Furthermore, since $T$ is compact, the net $\left\{n_{x_{i}}\left(s_{i}\right)\right\}_{i \in I}$ must cluster at some point $t \in T$. Since $\left\{x_{i}\right\}_{i_{\in} I}$ converges and $t \in \lim \sup \left[0, x_{i}\right]$, we have $x t=t$.

Fix $U \in B$ and $j \in I$ such that $x_{U} x=x_{U}$ for $i \geqslant j$. Let $J=\{i \in I: i \geqslant j\}$. Then $\left\{m_{x_{V} x_{i}}\left(s_{i}\right)\right\}_{i_{\in} J} \subseteq S_{x_{V}}$ and converges to $m_{x_{V} x}(s)$ in $S_{x_{V}}$ as a subset of $S^{\prime}$. Since the topology on $S_{x_{V}}$ as a subset of $S^{\prime}$ is the same as its original topology, this net must also converge to $m_{x_{V} x}(s)$ in $S_{x_{V}}$. Therefore the net $\left\{n_{x_{V}}\left(m_{x_{V} x_{i}}\left(s_{i}\right)\right)\right\}_{i \in J}$ converges to $n_{x_{V}}\left(m_{x_{V}}(s)\right)$ in $T$. Note that for each $i \geqslant j, n_{x_{V}}\left(m_{x_{V} x_{i}}\left(s_{i}\right)\right)=x_{U} n_{x_{i}}\left(s_{i}\right)$. Thus the net $\left\{x_{U} n_{x_{i}}\left(s_{i}\right)\right\}_{i_{\in} J}$ must converge to $n_{x_{V}}\left(m_{x_{V} x}(x)\right)=x_{U} n_{x}(s)$. Since $\left\{n_{x_{i}}\left(s_{i}\right)\right\}_{i_{\epsilon}} J$ clusters at $t$, we have that $x_{U} n_{x}(s)=x_{U} t$. Hence $x_{U} t=x_{U} n_{x}(s)$ for each $U \in B$ and $\left\{x_{U}\right\}_{U \in B}$ converging to $x$ imply $t=x t=x n_{x}(s)=n_{x}(s)$. Thus the net $\left\{n_{x_{i}}\left(s_{i}\right)\right\}_{i \in I}$ converges to $n_{x}(s)$ and $n$ is continuous.

A proposition similar to the following was stated and proved by Mislove (1969), p. 97 and that proof is still valid in this setting.

Proposition 3.6. Let everything be as in Propositions 3.3 and 3.5. Let $R$ be the relation on $S^{\prime}$ whose cosets are $R[s]=\left\{t \in S^{\prime}: n(s)=n(t)\right.$ and $\left.m_{x p(s)}(s)=m_{x p(t)}(t)\right\}$ where $x=p(s) p(t)$. Then $R$ is a closed congruence on $S^{\prime}$.

Definition 3.7. Let $(T, F)$ be a generalized pair and $S^{\prime}=\bigcup\left\{S_{x}: x \in X\right\}$ be the semigroup constructed in Proposition 3.3, $R$ be the congruence on $S^{\prime}$ defined in Proposition 3.6 and, finally, let $S(T, F)=S^{\prime} / R$. Then $S(T, F)$ is called the semigroup generated by the generalized pair $(T, F)$. 
Two of the main motivations of this work were, first, to generalize the construction of semigroups over trees, and second a desire to obtain a generalization of the following theorem.

THEOREM 3.8 (Mislove, 1974). Let $S$ be a compact semigroup. Then $\mathscr{H}$ is a congruence on $S$ and $S / \mathscr{H}$ is an abelian tree with idempotent endpoints if and only if $S=\mathscr{S}\left(T, X, S_{x}, m_{x y}, n_{x}\right)$ for some generalized collection $\left(T, X, S_{x}, m_{x y}, n_{x}\right)$.

The last section of this paper is devoted to the latter of these. As to the former, we now show that the construction given in this section does indeed generalize that of semigroups over trees. We first state a result that was announced by J. D. Lawson at the Second Florida Symposium on Automata and Semigroups at the University of Florida in April 1971.

THEOREM 3.9. Let $S$ be a semigroup on a tree. If $E(S)$ is a semilattice, then it is a Lawson semilattice.

THEOREM 3.10. Let $\left(T, X, S_{x}, m_{x y}, n_{x}\right)$ be a generalized collection. Then $(T, F)$ is a generalized pair where $F(x)=\left(S_{x}, n_{x}\right)$ and $F(y \hookrightarrow x)=\left(m_{x y}, h_{x y}\right)$ where $h_{x y}$ is such that $h_{x y} n_{y}=n_{x} m_{x y}$. Moreover, $S(T, F)$ is identical, algebraically and topologically, to $\mathscr{S}\left(T, X, S_{x}, m_{x y}, n_{x}\right)$.

Proof. Since $T$ is an abelian tree with idempotent endpoints, it is also a generalized tree with idempotent endpoints, and by Theorem $3.9 X=E(T)$ is a Lawson semilattice. Furthermore, from the definition of a generalized collection (see Mislove, 1974) it is clear that $F: X \rightarrow \mathscr{C} \times \mathscr{M}$ defined as above is such that $(T, F)$ is a generalized pair.

Let $S^{\prime}$ denote the semigroup constructed in Proposition 3.3 and $S_{M}$ denote the corresponding semigroup constructed from the generalized collection (see Mislove, 1974).

Define $i: S^{\prime} \rightarrow S_{M}$ by $i(s)=s$. Let $i(s) \in W(U, z, V)$ a basic open set in $S_{M}$. Here $U$ is a connected open set in $T$ containing $z \in X$ and $V$ is open in $S_{z}$. We consider two cases and the forms that $W(U, z, V)$ must have in each case.

Case $1, z$ is isolated in $z X$. Then by definition

$$
W(U, z, V)=\left\{t \in S_{M}: p(t) \in U, z p(t)=z \text { and } m_{z p(t)}(t) \in V\right\} .
$$

Clearly there is an open set $O$ in $X$ contained in $U$ with $z \in O$ and $z=\inf O$, since $X$ is a Lawson semilattice. Hence $W(0, V)$ is a basic open set in $S^{\prime}$ containing $S$ and it is trivial to show that $i(W(0, V)) \subseteq W(U, z, V)$.

Case 2, $z$ is not isolated in $z X$. In this case

$$
W(U, z, V)=\left\{t \in S_{M}: z \in[0, p(t)) \cap U, m_{z p(t)}(t) \in V\right\} .
$$


Since $Q=\{y \in X: z \in[0, y)\}$ is open in $X$ (see Ward, 1954), there is an open semilattice $U_{1}$ of $X$ containing $p(s)$ with $U_{1} \subseteq Q \cap U$. Let $V_{1}=m_{\varepsilon u}^{-1}(V)$ which is open in $S_{u}$, where $u=\inf U_{1}$. Hence $W\left(U_{1}, V_{1}\right)$ is open in $S^{\prime}$ containing $p(s)$, and if $t \in W\left(U_{1}, V_{1}\right)$, then $p(t)$ is in $U_{1} \subseteq Q \cap U$ and $m_{u p(t)}(t) \in m_{z u}^{-1}(V)$. Whence $m_{z p(t)}(t)$ is in $V$, that is, $t \in W(U, z, V)$ and $i\left(W\left(U_{1}, V_{1}\right)\right)$ is contained in $W(U, z, V)$.

From the above, $i$ is a continuous one-one map of the compact space $S^{\prime}$ onto the Hausdorff space $S_{M}$, that is, $i$ is a homeomorphism and $S^{\prime}=S_{M}$. Since the same congruence $R$ is employed in both instances, we have that $S(T, F)=S^{\prime} / R$ and $S^{\prime} / R=S_{M} / R=\mathscr{S}\left(T, X, S_{x}, m_{x y}, n_{x}\right)$.

\section{Characterization of $\mathscr{M} \mathscr{L}$-semigroups}

The main result of the paper appears in this section. It is the characterization of those compact semigroups $S$ on which $\mathscr{H}$ is a concruence, $S / \mathscr{H}$ is an abelian generalized tree with idempotent endpoints, and $E(S / \mathscr{H})$ is a Lawson semilattice. In particular we show that any such semigroup $S$ is the semigroup $S(S / \mathscr{H}, F)$ generated by the generalized pair $(S / \mathscr{H}, F)$, where $\Pi_{\mathscr{E}} F(x)$ is a well-chosen cylindrical subsemigroup of $S$ for each $x$ in $E(S / \mathscr{H})$, and $\Pi_{\mathscr{C}} F(y \rightarrow x)$ is translation by $1_{x}$. Conversely, if $S=S(T, F)$, then $\mathscr{H}$ is a congruence on $S$ and $S / \mathscr{H} \simeq T$.

Definition 4.1. A compact semigroup $S$ is a semigroup over a generalized tree, hereafter called an $\mathscr{M} \mathscr{L}$-semigroup, if $\mathscr{H}$ is a congruence on $S$ and $S / \mathscr{H}$ is an abelian generalized tree with idempotent endpoints and $E(S / \mathscr{H})$ is a Lawson semilattice. The category of $\mathscr{M} \mathscr{L}$-semigroups and continuous homomorphisms will be denoted by $\mathscr{M} \mathscr{L}$.

The proof of the following proposition is a straightforward modification of the analogous result by Mislove (1969), pp. 128-130 and as such its proof is omitted.

Proposition 4.2. If $(T, F)$ is a generalized pair, then $S(T, F)$ is an object of the category $\mathscr{M} \mathscr{L}$.

DEFINITION 4.3. Let $\mathscr{G}$ be the collection of all generalized pairs $(T, F)$. Let $(T, F)$ and $\left(T^{\prime}, G\right)$ be elements of $\mathscr{G}$. Then the pair $(e, w)$ is a morphism from $(T, F)$ to $\left(T^{\prime}, G\right)$ if $e: T \rightarrow T^{\prime}$ is a homomorphism and $w$ is a natural transformation from $\Pi_{\mathscr{Q}} F$ to $\Pi_{\mathscr{C}} G e$ which satisfies for each $x$ in $X, e\left(n_{x}(s)\right)=n_{e(x)}\left(w_{x}(s)\right)$ for all $s$ in $S_{x}$, $n_{e(x)}=\Pi_{\mathscr{M}}(G e(x))$. Composition of morphisms is defined as

$$
\left(e^{\prime}, w^{\prime}\right) \circ(e, w)=\left(e^{\prime} \circ e, w^{\prime} e \circ w\right)
$$

if $(e, w):\left(T_{1}, F\right) \rightarrow\left(T_{2}, G\right)$ and $\left(e^{\prime}, w^{\prime}\right):\left(T_{2}, G\right) \rightarrow\left(T_{3}, K\right)$. Within this framework $\mathscr{G}$ is a category. 
Proposition 4.4. Let $(T, F)$ and $\left(T^{\prime}, G\right)$ be objects of $\mathscr{G}$ and $(e, w):(T, F) \rightarrow\left(T^{\prime}, G\right)$. Then the map $f: S(T, F) \rightarrow S\left(T^{\prime}, G\right)$ defined by $f([s])=\left[w_{p(s)}(s)\right]$ is a continuous homomorphism of $S(T, F)$ into $S\left(T^{\prime}, G\right)$ where [] denotes the image of the element under the corresponding congruence $R$, as defined in Proposition 3.6.

Proof. Let $S_{1}$ and $S_{2}$ be the compact semigroups constructed from $(T, F)$ and $\left(T^{\prime}, G\right)$, respectively, as in Proposition 3.3. Define the map $f^{\prime}: S_{1} \rightarrow S_{2}$ as follows:

$$
f^{\prime}(s)=w_{x}(s) \text { if } s \in S_{x} .
$$

By use of the same argument that Bowman (1971) uses in the proof of his Theorem $1.4, f^{\prime}$ can be shown to be a continuous homomorphism. To complete the proof of the present proposition, we show that if $(s, t) \in R_{1}$ on $S_{1}$, then $\left(f^{\prime}(s), f^{\prime}(t)\right) \in R_{2}$ on $S_{2}$, that is, $f$ as the map induced from $f^{\prime}$ is a continuous homomorphism. Let $(s, t) \in R_{1}$; thus $n_{p(s)}(s)=n_{p(t)}(t)$ and $m_{x p(s)}(s)=m_{x p(t)}(t)$, where $x=p(s) p(t)$. We show that $n_{e(p(s))}\left(w_{p(s)}(s)\right)=n_{e(p(t))}\left(w_{p(t)}(t)\right)$. By definition of $(e, w)$,

and

$$
n_{e(p(s))}\left(w_{p(s)}(s)\right)=e\left(n_{p(s)}(s)\right)=e\left(n_{p(t)}(t)\right)
$$

$$
e\left(n_{p(t)}(t)\right)=n_{e(p(t))}\left(w_{p(t)}(t)\right) .
$$

Further, since $w$ is a natural transformation from $\Pi_{\mathscr{C}} F$ to $\Pi_{\mathscr{C}} G e$, we have $m_{e(x) e(p(s))}\left(w_{p(s)}(s)\right)=m_{e(x) e(p(t))}\left(w_{p(t)}(t)\right)$. Hence $\left(w_{p(s)}(s), w_{p(t)}(t)\right) \in R_{2}$ and $f^{\prime}$ induces $f$.

The following proposition follows immediately from Propositions 4.4 and 4.2 and the definition of composition in the category $\mathscr{G}$.

Proposition 4.5. If $(T, F) \in \mathscr{G}, S(T, F)$ is the semigroup generated by $(T, F)$ and for $(e, w):(T, F) \rightarrow\left(T_{2}, G\right)$ in $G, f_{(e, w)}$ is the homomorphism from $S(T, F)$ to $S\left(T_{2}, G\right)$ given in Proposition 4.4, then the map $F$ from $\mathscr{G}$ into $\mathscr{M} \mathscr{L}$, given by $F(T, F)=S(T, F)$ and $F(e, w)=f_{(e, w)}$ is a functor.

NOTATION. In what follows $\Sigma$ will denote the universal compact solenoidal semigroup as defined and described by Hofmann and Mostert (1966), p. 74. We are now ready to consider the main theorem of this section.

THEOREM 4.6. A semigroup $S$ belongs to the category $\mathscr{M} \mathscr{L}$ if and only if $S \simeq \mathscr{F}(T, F)$ for some generalized pair $(T, F)$.

Proof. The sufficiency is just Propositions 4.2 and 4.4 and so we now show the necessity, which is, in the language of category theory, to show that $\mathscr{F}$ is a representative functor. 
Let $T=S / \mathscr{H}$ and $X=E(S / \mathscr{H})$. Then $T$ has a zero by Lemma 2.6. Let $X^{\prime}=\{x \in X \backslash\{0\}: x$ is isolated in $[0, x] \cap X\}$, and if $x \in X^{\prime}$, let $x^{\prime}=\sup ([0, x) \cap X)$.

Let $x \in X^{\prime}$ and consider $n^{-1}\left[x^{\prime}, x\right]$, where $n: S \rightarrow S / H$ is the natural map. By using Propositions 2.10 and 2.11 one can modify the techniques of Mislove (1974) to show that there is a homomorphism $\varphi ; \Sigma \times H_{x} \rightarrow n^{-1}\left[x^{\prime}, x\right]$ with

$$
n\left(\varphi\left(\Sigma \times H_{x}\right)\right)=\left[x^{\prime}, x\right] \text { and } \varphi((0,0), h)=h
$$

for each $h$ in $H_{x}$, where $H_{x}=n^{-1}(x)$. Choose one such homomorphism, and let $S_{x}=\varphi\left(\Sigma \times H_{x}\right)$.

If $x \notin X^{\prime}$, let $S_{x}=n^{-1}(x)=H_{x}$. If $x, y \in X$ with $x y=x$, let $m_{x y}: S_{y} \rightarrow S_{x}$ be defined by $m_{x y}(s)=l_{x} s$.

If $x \in X$, let $n_{x}=n \mid S_{x}$ and if $x, y \in X$ with $x y=x$, let $h_{x y}: n_{y}\left(S_{y}\right) \rightarrow n_{x}\left(S_{x}\right)$ be the map induced by $m_{x y}, n_{x}$ and $n_{y}$ so that $h_{x y} \circ n_{y}=n_{x} \circ m_{x y}$.

Now define $F: X \rightarrow \mathscr{C} \times \mathscr{M}$ by $F(x)=\left(S_{x}, n_{x}\right)$ and $F(y \hookrightarrow x)=\left(m_{x y}, h_{x y}\right)$ for each $x$ in $X$ and morphism $y \subset x$ in $X$.

By a straightforward modification of the corresponding proofs by Mislove (1974) it can be shown that $F$ is a functor satisfying all but condition (b) (iv) of the definition of a generalized pair. To accomplish this we make the following changes: for $x \in X$, let $F^{\prime}(x)=\left(\{x\} \times S_{x}, n_{x}^{\prime}\right)$ and $F^{\prime}(y \subset x)=\left(m_{x y}^{\prime}, h_{x y}^{\prime}\right)$, where if $x y=x$, $m_{x y}^{\prime}(y, s)=\left(x, m_{x y}(s)\right)$ and $n_{x}^{\prime}(x, s)=n_{x}(s)$. Clearly, $\left(T, F^{\prime}\right)$ is now a generalized pair.

We now show that $\mathscr{F}\left(T, F^{\prime}\right) \simeq S$. Let $S^{\prime}=\bigcup\left\{T_{x}: x \in X\right\}$, where $T_{x}=\{x\} \times S_{x}$ for each $x \in X$, be the semigroup constructed in Proposition 3.3 and define $f: S^{\prime} \rightarrow S$ by $f(x, s)=s$ for each $(x, s) \in S^{\prime}$. If we show that $f$ is a continuous surmorphism such that $f(x, s)=f(y, t)$ if and only if $((x, s),(y, t)) \in R$, the congruence defined in Proposition 3.6, then $\mathscr{F}\left(T, F^{\prime}\right)$ will be isomorphic to $S$ under the induced map.

The same proof utilized by Mislove (1974) will show that $f$ is an algebraic surmorphism and so we restrict ourselves to showing that $f$ is continuous.

Let $\left\{\left(x_{i}, s_{i}\right)\right\}_{i \in I}$ converge to $(x, s)$ in $S^{\prime}$. Then $\left\{s_{i}\right\}_{i \in I}$ is a net in $S^{\prime}$ and thus must cluster at some point $t \in S^{\prime}$. By choosing a subnet we may assume convergence. If $y_{i}=x x_{i}$ for each $i \in I$, then by possibly picking subnets, we have one of the following: either $y_{i} \neq x$ for each $i \in I$ or $y_{i}=x$ for each $i \in I$. Let $B$, the neighborhood system of open semilattices about $x$ in $X$, be directed by $U \leqslant V$ if and only if $V \subseteq U$. In the former case above, let $\left\{x_{U}\right\}_{U \in B}$ be a net in $X$ with $x_{U}=\inf U$ for each $U \in B$. Since $X$ is a Lawson semilattice this net converges to $x$ with $x_{U} x=x_{U}$ for each $U \in B$ and $x_{U} x_{V}=x_{U}$ if $U \leqslant V$. If $y_{i}=x$ for each $i \in I$, let $\left\{x_{U}\right\}_{U \in B}$ be the constant net $\{x\}$. Note that in either case for each $U \in B$, the set $\left\{i \in I: y_{i} x_{U}=x_{U}\right\}$ is a residual subset of $I$. Fix $U \in B$. Let $j \in I$ such that $y_{i} x_{U}=x_{U}$ if $i \geqslant j$ in $I$ and let $J=\{i \in I: i \geqslant j\}$. Then $\left\{m_{x_{U} x_{i}}\left(s_{i}\right)\right\}_{i_{\in} J} \subseteq S_{x_{V}}$ and converges to $m_{x_{V} x}(s)$ in $S_{x_{V}}$ as a subset of $S^{\prime}$. Moreover, $\left\{1_{x_{V}} s_{i}\right\}_{i_{\epsilon} J}$ converges to $1_{x_{D}} t$ in $S$ as multiplication is continuous in $S$. But $m_{x_{V} x_{i}}\left(s_{i}\right)=1_{x_{V}} s_{i}$ for each $i \in J$, and as $S_{x_{V}}$ is a closed subset of 
$S, 1_{x_{V}} t \in S_{x_{V}}$. By Lemma 2.4 the topology on $S_{x_{V}}$ as a subset of $S^{\prime}$ is the same as its topology as a subset of $S$, and hence $m_{x_{v} x}(s)=1_{x_{D}} t$. Since $U \in B$ was arbitrary and $1_{x_{V}} s=m_{x_{V} x}(s)$ for each $U \in B$,

$$
\begin{aligned}
s=1_{x} s & =\left(\lim _{U \in B} 1_{x_{V}}\right) s \\
& =\lim _{U \in B}\left(1_{x_{U}} t\right) \\
& =\left(\lim _{U \in B} 1_{x_{V}}\right) t \\
& =1_{x} t \\
& =t .
\end{aligned}
$$

Thus $\left\{f\left(\left(x_{i}, s_{i}\right)\right)\right\}_{i \in I}$ converges to $f(x, s)$ and $f$ is continuous.

All that remains to be shown is that the kernel congruence of $f$ is precisely the congruence $R$ defined in Proposition 3.6. If $f(x, s)=f(y, t)$, then it is easy to show that $((x, s),(y, t)) \in R$. Thus we suppose that $((x, s),(y, t))$ is in $R$ and show that $f(x, s)=f(y, t)$. Since $((x, s),(y, t)) \in R, n(s)=n_{x}^{\prime}(x, s)=n_{y}^{\prime}(y, t)=n(t)$. But $1_{x} s=s$, $1_{y} t=t$ and thus $1_{y} s=s$ and $1_{x} t=t$ since $s$ and $t$ are $\mathscr{H}$-related. If $z=x y$, then $s=1_{x} 1_{y} s=1_{z} s=m_{z x}(s)=m_{z y}(t)=1_{z} t=1_{x} 1_{y} t=t$ and thus $f(x, s)=f(y, t)$.

Hence $\mathscr{F}\left(T, F^{\prime}\right) \simeq S$ by the isomorphism induced by $f$ and we have completed the proof of the theorem.

The results of this paper stemmed from a desire to obtain at least a partial solution to Problem P5 of Hofmann and Mostert (1966) and this we have done.

\section{REFERENCES}

T. T. Bowman (1971), "A construction principle and compact Clifford semigroups", Semigroup Forum 2, 343-353.

H. Cohen and I. S. Krule (1959), "Continuous homomorphic images of real clans with zero", Proc. Amer. Math. Soc. 10, 106-109.

K. H. Hofmann and P. S. Mostert (1966), Elements of Compact Semigroups (Charles Merrill, Columbus).

R. P. Hunter (1959), "On the semigroup structure of continua", Trans. Amer. Math. Soc. 93, 356-368.

R. P. Hunter (1961), “On a conjecture of Koch”, Proc. Amer. Math. Soc. 12, 138-139.

J. L. Kelley (1955), General Topology (Van Nostrand Company Inc., Princeton, New Jersey).

R. J. Koch (1957), "Note on weak outpoints in clans", Duke Math J. 24, 611-616.

R. J. Koch and I. S. Krule (1960), "Weak cutpoint ordering on hereditarily unicoherent continua", Proc. Amer. Math. Soc. 11, 679-681.

M. W. Mislove (1969), "Four problems about compact semigroups", Dissertation, The University of Tennessee, Knoxville.

M. W. Mislove (1974), "Semigroups over trees", Trans. Amer. Math. Soc. 195, 383-400.

B. Mitchell (1965), Theory of Categories (Academic Press, New York, 1965).

R. C. Phillips (1963), "Interval clans with nondegenerate kernel", Proc. Amer. Math. Soc. 14, $396-400$. 
L. E. Ward (1954), "A note on dendrites and trees", Proc. Amer. Math. Soc. 5, 992-994.

L. E. Ward (1957), “Mobs, trees, and fixed points”, Proc. Amer. Math. Soc. 8, 798-804.

L. E. Ward, Jr. (1958), "On dendritic sets", Duke Math. J. 25, 505-514.

The Ohio State University

Newark, Ohio

U.S.A. 